NASA/TM-2001-210821

(4)

Rayleigh Scattering Diagnostic for Dynamic Measurement of Velocity Fluctuations in

High Speed Jets

Richard G. Seasholtz

Glenn Research Center, Cleveland, Ohio

Jayanta Panda

Ohio Aerospace Institute, Brook Park, Ohio

Kristie A. Elam

Akima Corporation, Fairview Park, Ohio

Prepared for the

39th Aerospace Sciences Meeting and Exhibit

sponsored by the American Institute of Aeronautics and Astronautics

Reno, Nevada, January 8-11, 2001

National Aeronautics and

Space Administration

Glenn Research Center 
Available from

NASA Center for Aerospace Information 7121 Standard Drive

Hanover, MD 21076

Price Code: A03

National Technical Information Service 5285 Port Royal Road Springfield, VA 22100 Price Code: A03

Available electronically at http://gltrs.grc.nasa.gov/GLTRS 


\title{
RAYLEIGH SCATTERING DIAGNOSTIC FOR DYNAMIC MEASUREMENT OF VELOCITY FLUCTUATIONS IN HIGH SPEED JETS
}

\author{
Richard G. Seasholtz \\ National Aeronautics and Space Administration \\ Glenn Research Center \\ Cleveland, Ohio 44135 \\ Jayanta Panda \\ Ohio Aerospace Institute \\ Brook Park, Ohio 44142 \\ Kristie A. Elam \\ Akima Corporation \\ Fairview Park, Ohio 44126
}

\begin{abstract}
A flow diagnostic technique based on the molecular Rayleigh scattering of laser light is used to obtain dynamic density and velocity data in a high speed flow. The technique is based on analyzing the Rayleigh scattered light with a Fabry-Perot interferometer used in the static, imaging mode. An analysis is presented that established a lower bound for measurement uncertainty of about $20 \mathrm{~m} / \mathrm{sec}$ for individual velocity measurements obtained in a $100 \mu \mathrm{sec}$ time interval. Software and hardware interfaces were developed to allow computer control of all aspects of the experiment and data acquisition. The signals from three photomultiplier tubes were simultaneously recorded using photon counting at a $10 \mathrm{kHz}$ sampling rate and 10 second recording periods. Density and velocity data, including distribution functions and power spectra, taken in a Mach 0.8 free jet are presented.
\end{abstract}

\section{INTRODUCTION}

In this paper we describe a flow diagnostic based on the molecular Rayleigh scattering of laser light for use in NASA test facilities. This diagnostic will provide dynamic aerothermodynamic data that are not presently available. These data will be important in studies such as the investigation of growth and decay of turbulent fluctuations. The effort is part of the non-intrusive instrumentation development program supporting propulsion research at the NASA Glenn Research Center. This work is directed to the measurement of fluctuations in flow velocity, density, and temperature for jet noise studies. One of the main objectives in jet noise research is to identify noise sources in the jet and to determine their contribution to noise generation. In particular, researchers have focused on the correlation of fluctuations in flow parameters with far field noise ${ }^{l}$.

A variety of nonintrusive, laser based flow diagnostics (Rayleigh scattering, Laser Doppler Velocimetry (LDV), Particle Imaging Velocimetry (PIV), Laser Induced Fluorescence (LIF)) are routinely being applied for time average and instantaneous planar measurements of velocity, density, temperature, and species concentrations. However, an important class of measurements, high frequency response dynamic measurements of flow parameters, is not addressed by current laser diagnostics. This type of time history data is needed to determine, for example, density and velocity spectra, density-velocity correlations, and two-point correlations. Although LDV can typically achieve data rates of a few tens of $\mathrm{kHz}$, it is difficult to achieve much higher rates without introducing larger amounts of seed material into the flow. In practice, LDV normally provides mean velocity and a measure of turbulence intensity. Furthermore, the measurements are random in time, which makes it difficult to obtain time history data needed for power spectra. In turbulent flows, LDV measurements are beset by a variety of so-called biasing errors, caused by correlations between the measurement rate and flow properties. Planar techniques, such as PIV and planar Rayleigh scattering, provide a large number of simultaneous measurements in a plane, but are generally limited to low sampling rates, determined by the pulse repetition rate of the laser and by the time needed to transfer image data from the camera. New techniques are needed to provide nonintrusive, dynamic measurements that can provide data similar to that provided by hot wire anemometers. Since it is unrealistic to expect (at least at the present time) to make measurements at a large number of locations and at a high 
sampling rate, it seems prudent to develop a laser diagnostic capable of point measurements at high sampling rates.

Because the Rayleigh scattering technique under study is based on molecular scattering rather than particle scattering, no seed material need be injected into the flow. One difficulty with LDV and PIV is that they require the flow to be "seeded" with micron size seed particles to provide a sufficient concentration of scattering centers. The reliance on seed particles presents a number of difficulties, such as difficulty injecting a uniform cloud of seed, and providing seed material that can withstand the flow environment. For high temperatures, refractory seed materials are necessary. In addition, seed material can contaminate the facility, coating surfaces and windows. Facility engineers are often wary of the introduction of this foreign, often abrasive material, into their equipment. A further limitation of particle scattering methods is that the particles, although small, may not be able to follow large flow accelerations, thus introducing inaccuracy in the prediction of the gas velocity.

These problems associated with particle scattering measurements are eliminated if molecular scattering is used, since the gas molecules that constitute the flow under study are used as the scattering centers. The simplest molecular scattering based diagnostic is Rayleigh scattering. The frequency spectrum of Rayleigh scattering is closely related to the velocity distribution of the scattering gas. The spectrum may be analyzed to determine temperature, density, and velocity. Density is simply proportional to the total scattered light; temperature is related to the width of the Rayleigh spectrum; and one component of velocity is proportional to the shift of the spectral peak from the frequency of the incident light. Because the spectral width is also a function of the molecular weight of the gases in the flow, knowledge of the gas composition is generally required; however, this is not a concern in the proposed work, which is directed toward air flows where the composition is well defined. In any case, velocity measurements, because they are determined from the frequency of the peak of the spectrum, are independent of the gas composition. Rayleigh scattering is particularly suitable for measurement of supersonic and hypersonic velocity where the mean molecular velocity (flow velocity) is larger than the random molecular velocity (temperature).

Because of the relative simplicity of Rayleigh scattering based gas density measurements, they have been more widely used than velocity and temperature measurements. In our work, for example, time and phase averaged density measurements have been made in an underexpanded supersonic free jet in support of jet noise studies $^{2}$. We have also demonstrated simultaneous multiple point density measurements for determining gas density fluctuation spectra, cross-spectra, and cross correlation functions in a low speed heated jet ${ }^{3}$. The more difficult problem of dynamic velocity and temperature measurements was addressed in two previous papers. The first paper ${ }^{4}$ used direct imaging of the Rayleigh scattered light through a Fabry-Perot interferometer. One photomultiplier tube (PMT) was used to detect the total Rayleigh scattered light for density measurements (as is done in the present paper). Two additional PMT's were used to determine the velocity and temperature. A disadvantage of this method, however, is that it required the detection system, including the sensitive Fabry-Perot interferometer, to be located close to the experiment. A second paper ${ }^{5}$ described a system that used an optical fiber to transmit scattered light from the experiment to the Fabry-Perot Interferometer (FPI), which could be located at some distance from the experiment. This allowed the interferometer to be located in a controlled environment, where the temperature can be held constant and where acoustic noise and vibration can be minimized. This system also incorporated an $8 \times 8$ multiple anode PMT that was used to detect the interference pattern formed by the FPI. However, the quantum efficiency of the $8 \times 8$ multiple anode photomultiplier was only about $5 \%$. This is low compared with the quantum efficiency of about $25 \%$ that can be achieved with single PMT's. In addition, both these systems used analog data acquisition equipment. which resulted in relatively high noise levels in the detected signals.

In this paper we present an improved version of these systems. The present system uses three $25 \%$ quantum efficiency PMT's to improve the detection efficiency. A new photon counting data acquisition system is used, which improves the signal to noise ratio of the detected signals by reducing the amount of noise due to electrical interference. Also, new computer software and hardware have been incorporated that greatly simplify the system operation. Some basic background material related to molecular Rayleigh scattering based diagnostic systems is first presented. The lower bounds for measurement of density, temperature, and velocity are given, assuming errors result only from Poisson noise generated in the photo detection process. This establishes the best possible measurements that could only be approached by use of an ideal instrument. We then describe the scheme used to measure dynamic velocity based on a Fabry-Perot interferometer and three PMTs. The lower bounds for velocity measurement uncertainties are calculated for this particular system.

An experiment conducted to evaluate the technique on a subsonic flow (up to about $300 \mathrm{~m} / \mathrm{sec}$ ) is then described. Results are given for data sampled at a $10 \mathrm{kHz}$ 
rate. Examples of velocity time history and power spectrum of velocity fluctuations are given.

\section{$\underline{\text { Rayleigh scattering }}$}

\section{THEORY}

The spectrum for Rayleigh scattering from a low density gas has a Gaussian profile given by

$$
S\left(f-f_{o}\right) d f=\frac{2 \sqrt{\pi}}{a K} \exp \left\{-\left[\frac{2 \pi\left(f-f_{o}\right)-\mathbf{K} \cdot \mathbf{u}}{a K}\right]^{2}\right\} d f
$$

where $f_{\mathrm{o}}$ is the laser frequency and $\mathbf{u}$ is the mean gas velocity. The interaction wave vector is $K=\mathbf{k}_{\mathrm{s}}-\mathbf{k}_{\mathrm{o}}$ (with $\mathbf{k}_{\mathbf{v}}$ and $\mathbf{k}_{\mathbf{s}}$ being the wave vectors of the incident and scattered light), and $a=(2 \kappa T / m)^{1 / 2}$ is the most probable molecular speed (with $\kappa$ being Boltzmann's constant, $m$ the molecular mass, and $T$ the gas temperature). Note that the spectral peak is shifted by a frequency proportional to the component of the bulk velocity in the $\mathbf{K}$ direction. The spectral width is proportional to the square root of the gas temperature. It is convenient to introduce the velocity component $u_{K}=\mathbf{K} \cdot \mathbf{w} / K$, which represents the measured velocity component.

The assumption of a Gaussian shaped Rayleigh scattering spectrum is only valid if

$$
y=\frac{p}{\eta K a} \ll 1
$$

where $p$ is the gas pressure and $\eta$ is the shear viscosity. Collective effects of the molecular motions become important for higher density gases $(y \sim 1)$ and a more detailed kinetic theory model, such as the Tenti S6 model ${ }^{6}$, is required to describe the Rayleigh scattering spectrum. Although the Tenti spectrum does differ significantly from a Gaussian spectrum for the flows we are studying, similar results are obtained for the uncertainty analysis we conducted. We, therefore, used the Gaussian spectral model to reduce the computational time.

\section{Lower bounds for ideal measurements}

Since Rayleigh scattering is a relatively weak process, the uncertainty in the measurements often is set by the photon statistical noise (shot noise), which determines the lower bound on measurement uncertainty. For example, the variance in the number of photoelectron counts for a Poisson process is equal to the mean number of counts. Thus the lower bound for the relative uncertainty in the measurement of gas density $\rho$, is equal to the square root of the variance divided by the mean counts $N_{R}$. The lower bounds for temperature and velocity uncertainties have also been evaluated for a low-density, one-component gas. The relative uncertainties caused by photon statistics for this case can be written ${ }^{7.8}$.

$$
\frac{\sigma(\rho)}{\rho}=\left(\frac{1}{N_{R}}\right)^{1 / 2}, \quad \frac{\sigma(T)}{T}=\left(\frac{2}{N_{R}}\right)^{1 / 2}, \quad \sigma\left(u_{K}\right)=\frac{a}{\left(2 N_{R}\right)^{1 / 2}}
$$

These relations provide a lower bound for the measurement uncertainties, which can only be attained if the shot noise is the dominant noise and if an ideal instrument is used.

Consider an experiment with the following parameters: a laser $(532 \mathrm{~nm})$ with output power $P_{o}=$ $5 \mathrm{~W}$, air at $\operatorname{STP}(\mathrm{T}=293 \mathrm{~K}, \mathrm{p}=1 \mathrm{~atm})$, probe volume length $L_{x}=1 \mathrm{~mm}, \mathrm{f} / 4$ collecting optics (i.e., solid collection angle $\Omega=0.05 \mathrm{sr}$ ), and efficiency factor $\varepsilon=$ $5 \%$. The rate of detected photons, given by

$$
\begin{aligned}
& N_{R}=\frac{\varepsilon P_{o} n L_{x} \lambda \Omega}{h c}\left(\frac{\mathrm{d} \sigma}{\mathrm{d} \Omega}\right) \sin ^{2} \chi \\
& =50 \text { million counts } / \mathrm{sec}
\end{aligned}
$$

In this equation, $n$ is the gas number density, $d \sigma / d \Omega$ is the differential scattering cross section $\left(6.13 \times 10^{-32} \mathrm{~m}^{2} / \mathrm{sr}\right.$ for air at $532 \mathrm{~nm}), \chi$ is the angle between the electric field vector of the (linearly polarized) incident light and the direction of the scattered light, $h$ is Planck's constant, and $c$ is the velocity of light. If we wish to obtain independent measurements at a $10 \mathrm{kHz}$ rate, the total number of detectable photons in each period would be 5000. Equation 2 gives the lower bound for measurement of density, temperature and velocity for each period:

$$
\frac{\sigma(\rho)}{\rho}=1.4 \%, \quad \frac{\sigma(T)}{T}=2.0 \%, \quad \sigma\left(u_{K}\right)=4.1 \mathrm{~m} / \mathrm{sec}
$$

This shows that high sampling rate Rayleigh scattering measurements are at least feasible. It must be emphasized, however, that these values represent the best possible measurements given an ideal instrument. In practice, we are limited to instruments, such as the Fabry-Perot interferometer used here, which result in significantly higher uncertainties, as described below.

\section{Lower bounds for practical instrument}

Estimates of the measurement uncertainty for the technique described here, where the Rayleigh scattered light is analyzed with a planar mirror Fabry-Perot interferometer, are obtained by numerically calculating the Cramer-Rao lower bound ${ }^{9}$. The variance of the estimate of a parameter $\alpha_{i}$ (e.g., temperature or velocity) is given by 


$$
V\left(\alpha_{i}\right)=\left[\Gamma^{-1}\right]_{i i}
$$

where no summation over repeated indices is implied. For Poisson statistics, $\Gamma$ is the Fisher information matrix with elements

$$
\Gamma_{i j}=\sum_{q} \frac{1}{\left\langle N_{D_{q}}\right\rangle} \frac{\partial\left\langle N_{D_{q}}\right\rangle}{\partial \alpha_{i}} \frac{\partial\left\langle N_{D_{q}}\right\rangle}{\partial \alpha_{j}}
$$

where $\left\langle N_{D q}\right\rangle$ is the expected number of counts from the $q^{\text {th }}$ photodetector. The summation is over the number of photodetectors, and the quantities $\left\langle\mathrm{N}_{D_{q}}\right\rangle$ depend on the details of the optical system and the flow parameters, as presented below in the Uncertainty analysis section.

\section{Optical configuration}

In order to perform an uncertainty analysis, we first describe the setup (fig. 1) used for the experimental work. Light from a $5 \mathrm{~W}, 532 \mathrm{~nm}$, single-frequency, $\mathrm{Nd}$ :Vanadate $\mathrm{CW}$ laser was focused by lens $\mathrm{Ll}$ (350 $\mathrm{mm}$ focal length) to a $150 \mu \mathrm{m}$ diameter beam at the probe volume. The laser beam was terminated in light trap LT. Rayleigh scattered light is collected at a $90^{\circ}$ scattering angle and focused by L2 (two $160 \mathrm{~mm}$ focal length lenses) into a $1 \mathrm{~mm}$ core diameter, $20 \mathrm{~m}$ long optical fiber. The light exiting the fiber is collimated by lens $\mathrm{L} 3$ (145 mm focal length) and split into two paths with an uncoated optical flat (BS1). About $10 \%$ of the light is reflected and focused by lens L4 (85 mm focal length) onto PMT 1 (quantum efficiency $\sim 25 \%$ ). This signal is proportional to gas density. The light transmitted by the beamsplitter is directed through a planar mirror Fabry-Perot interferometer $(70 \mathrm{~mm}$ dia. mirrors, $90 \%$ reflectivity, $10 \mathrm{GHz}$ free spectral range (FSR), finesse $\sim 15$ ). The light exiting the interferometer is focused by the fringe forming lens, L6. This lens consists of a pair of lenses (f/2 $135 \mathrm{~mm}$ focal length and $\mathrm{f} / 1.250 \mathrm{~mm}$ focal length) that has an effective focal length of $1600 \mathrm{~mm}$.

At the focal plane of the fringe pattern, the light is divided into two parts by a pair of mirrors (image dissector). A small mirror $(6 \mathrm{~mm}$ dia.) is centered on the fringe pattern and directs light from the central part of the fringe through lens $\mathrm{L} 7(100 \mathrm{~mm}$ focal length) to PMT 2. This small mirror is mounted on a larger mirror ( $25 \mathrm{~mm}$ dia.) that directs light from the outer part of the fringe through $\mathrm{L} 8(100 \mathrm{~mm}$ focal length) to PMT 3. The mirrors are tilted $\pm 3^{\circ}$ with respect to the optical axis. A typical calculated image of the inner fringe of Rayleigh scattered light is shown in figure 2. Note that a flow in the direction of the $\mathbf{K}$ vector, which here is in the jet flow direction (fig. 1), results in a positive frequency shift and increasing fringe diameter, while a flow in the direction opposite the $\mathbf{K}$ vector results in a negative frequency shift and decreasing fringe diameter. Thus, as the frequency of the Rayleigh scattered light increases, less light is detected by PMT 2 and more light is detected by PMT 3. At the mirror location, the diameter of the image of the optical fiber is $11 \mathrm{~mm}$. Additional optics were included to provide a reference image of light at the unshifted laser frequency. To accomplish this, several components could be placed in the optical path using remotely controlled pneumatic actuators. When placed in the beam path, mirror $M$ directed laser light onto the diffuser (D) that scattered light into the optical fiber. Also, a prism assembly (PA) could be placed in the light path between the Fabry-Perot interferometer and the fringe forming lens. This served to direct light into a standard video camera. The video signal from this camera was digitized by a PC frame grabber card. A computer program analyzed this image and generated signals to control the Fabry-Perot mirror alignment.

\section{Uncertainty analysis}

We can now calculate the Cramer-Rao lower bounds given by equation 4 for velocity measurements based on the optical configuration described in the previous section. This allows us to conduct parametric studies to determine the optimum configuration for the Fabry-Perot interferometer and for the light detection system. The expected number of photons detected in time interval $\Delta t$ by detector 1 (PMT 1 ) is simply $R_{B} N_{R} \Delta t$ where $R_{B}$ is the reflectivity of the beamsplitter BS and $N_{R}$ is given by equation 3 . The expected number of photons detected by PMT 2 and PMT 3 are

$<N_{D q}>=\left(1-R_{B}\right) N_{R} \Delta t \iint S_{R}(f) I_{F P}\left(f, \theta_{r}\right) f_{L}^{2} d f d A$

where the integrations are over frequency and the area of the $q^{\text {th }}$ detector (i.e., PMT 2 or 3 ), $f_{L}$ is the focal length of the fringe forming lens, and $I_{F P}$ is the FabryPerot instrument function given by ${ }^{10}$

$$
I_{F P}(\psi)=\left[1+F \sin ^{2}\left(\frac{\psi}{2}\right)\right]^{-1}
$$

where $\psi$ is the phase change (neglecting any phase change on reflection) of the light between successive reflections given by

$$
\psi\left(f, \theta_{r}\right)=\frac{4 \pi f \mu d \cos \theta_{r}}{c}
$$


Here, $\mu$ is the refractive index of the medium in the Fabry-Perot cavity, $d$ is the Fabry-Perot mirror spacing, $\theta_{r}$ is the angle between the ray and the optic axis, and $F=1 /\left(\sin ^{2}\left(\pi / 2 N_{E}\right)\right.$ where $N_{E}$ is the effective finesse. In general, the image of a monochromatic extended source located in the object plane consists of a series of unequally spaced concentric rings. In this work, however, the field of view is restricted by the diameter of the optical fiber and includes only the inner fringe as shown in figure 2 .

We can readily calculate the lower bounds for density, velocity, and temperature measurement uncertainties based on the detected light using three PMT's as shown in figure 1. The uncertainties are evaluated as a function of the zero velocity fringe order $n_{o}$ and the flow velocity.

It is convenient to describe the fringe location in terms of the fringe order rather than fringe radius because of the nonlinear nature of the spectral response of the Fabry-Perot. For example, if there is a bright fringe on axis, concentric bright fringes occur at integral values of lower orders, but the change in fringe radius decreases with decreasing fringe order.

Here we define the fractional order of the fringe with radius $r$ as

$$
n=\frac{d}{\lambda}\left(\frac{r}{f_{L}}\right)^{2}
$$

For example, at the location of the image dissector, the fringe order of the $6 \mathrm{~mm}$ diameter mirror is 0.10 . Likewise, the fringe order corresponding to the diameter of the image of the optical fiber is 0.33 . And, if unshifted laser light generates a bright fringe with radius $r_{o}$, we refer to this as order $n_{o}$. Note that here we are using the term "fringe order" to denote change of the fringe order from the actual fringe order on the optical axis, which is $2 d / \lambda$

frequency is

The change in fringe order with optical

$$
\Delta n=\frac{\Delta f}{F S R}
$$

so the change of fringe order $\Delta n$ with change of velocity $\Delta V$ is given by

$$
\Delta n=\frac{(2 / \lambda) \sin \left(\theta_{s} / 2\right)}{F S R} \Delta V
$$

where $\theta_{s}$ is the scattering angle. For example, with a velocity of $100 \mathrm{~m} / \mathrm{sec}$, a FSR of $10 \mathrm{GHz}$ and $90^{\circ}$ degree scattering, the change in the fringe order $\Delta n$ is 0.0266 .

The lower bound for velocity uncertainty was numerically evaluated as a function of the fringe order $n_{0}$ and the velocity using equations $1,3,4$, and 6 . For these calculations, either two or three unknown parameters were assumed (i.e., the $\alpha_{i}$ in eq. 5 were $N_{R}$, and $u_{k}$, or $N_{R}, u_{k}$, and $T$ ). The results are shown in figure 3 for the optical system described above for a single measurement using $0.5 \mathrm{~mJ}$ of laser energy (i.e, a measurement made in a $100 \mu \mathrm{sec}$ time interval using a $5 \mathrm{~W}$ laser). Note that the minimum uncertainty for velocity measurements depends on the fringe order $n_{0}$ and velocity. Thus, the fringe order should be set to minimize the velocity uncertainty for the range of velocities to be measured. The mean number of photoelectron counts in a $100 \mu \mathrm{sec}$ time interval for the three PMT's are shown in figure 3a. Two cases of the lower bound of velocity uncertainty are shown for temperatures ranging from 150 to $300 \mathrm{~K}$, where the density is fixed. One is for a flow with unknown temperature (fig. 3b), and one is for a flow with known temperature (fig. 3c). Note that, as expected, the measurement uncertainty is lower for colder gas. Also, in general, knowing the temperature significantly decreases the predicted measurement uncertainty. In this example, the uncertainty for a flow with known temperature is about $25 \mathrm{~m} / \mathrm{sec}$ for a $300 \mathrm{~K}$ gas. As discussed above, an ideal measurement taken for a $100 \mu \mathrm{sec}$ time interval would have an uncertainty of about $4 \mathrm{~m} / \mathrm{sec}$. Thus, for our FPI based measurement system, the expected velocity uncertainty is about a factor of six larger than the uncertainty that would be obtained with an ideal instrument. Finally, an important observation is that a low velocity measurement uncertainty can be expected over a $600 \mathrm{~m} / \mathrm{sec}$ velocity range (for the more general case of unknown temperature).

\section{Setup}

\section{EXPERIMENT}

The optical system described above and shown in figure 1 (with the laser output power equal $5 \mathrm{~W}$ ) was used to measure the flow in a subsonic free jet $(8.8 \mathrm{~mm}$ exit diameter). The probe volume was located in the potential core of the jet to avoid any dust particles in the ambient air. The laboratory compressed air supply used for the jet was passed through a three-stage filter to remove particles and oil vapor. As shown in figure 1 , the jet axis bisected the incident and scattered light directions. With this configuration, the measured velocity component was the jet axial component. Since the flow velocity was in the same direction as the $\mathbf{K}$ vector, the frequency shift was positive. Thus the Fabry-Perot interferometer fringe diameter of the Rayleigh scattered light increased as the flow velocity increased. An electronic pressure gauge was used to measure the total pressure in the nozzle plenum. These pressure measurements were used to calculate the flow velocity and temperature using the isentropic flow relations. A high precision electronic thermometer was 
used to monitor the temperature within the FPI enclosure to correlate interferometer stability with temperature.

Data Acquisition

A flow chart and block diagram of the PC based control and data acquisition system developed for this work is shown in figure 4.

The photoelectron pulses from the three PMT's were amplified (Gain $=5$ ) and sent to constant fraction discriminators (CFD). The CFD's output $10 \mathrm{~ns}$ wide TTL level pulses that were counted by a PC countertimer board. Pulses could be simultaneously counted on the three channels at rates to $80 \mathrm{MHz}$ for indefinite times. Typical photoelectron count rates for this work were on the order of $5 \mathrm{MHz}$. The accumulated counts on each channel were recorded at preset time intervals, typically $100 \mu \mathrm{sec}$, and stored on the computer hard disk. The number of counts in each time interval was given by the difference between adjacent values of the accumulated counts.

The flow chart of the software used to control all aspects of the instrument system operation is shown in figure $4 \mathrm{a}$ and the block diagram of the hardware is shown in figure $4 \mathrm{~b}$. The system had two modes of operation: (a) the Reference mode, where the automatic Fabry-Perot interferometer stabilization is active; and (b) the Rayleigh mode, where the Rayleigh scattering data is acquired. When the program is run, the sequence of operations shown in figure $4 \mathrm{a}$ is executed. The operator has the option of either switching to the Reference mode between each data set or taking sequential data sets without switching to the reference mode. The advantage of using the Reference mode between data sets is that any drift in the interferometer is minimized.

\section{Data processing}

The gas density can be obtained in a straightforward manner, since it is proportional to the total Rayleigh scattered light measured by PMT 1 . Calibration constants (slope and intercept) are found from a linear least squares fit (shown on fig. 5) of the PMT 1 counts plotted as a function of the gas density.

One approach to processing the PMT signals in order to obtain the velocity would be to use parameter estimation techniques based on the model developed for the calculation of the lower bounds of measurement uncertainty. This would entail doing a nonlinear fit of the data to the model function given by equation 6 . However, because of the large quantity of data (acquired at a $10 \mathrm{kHz}$ rate for the three PMT's) a simpler procedure was used. This procedure took advantage of the fact that the temperature only varied over a small range, from about $295 \mathrm{~K}$ for low flow to about $255 \mathrm{~K}$ for the maximum flow velocity of about $280 \mathrm{~m} / \mathrm{sec}$. The operating region was selected so the count rate for the inner and outer regions were approximately linear with respect to velocity. The first step was to obtain a set of mean data for the velocity range of interest, where the velocity was calculated from the total temperature and pressure ratio using isentropic flow relations. A linear fit was then done to obtain the four constants A1, B1, A2, and B2 as shown for a typical data set on figure 6 .

$$
I=A_{1}+B_{1} V, \quad O=A_{2}+B_{2} V
$$

These are solved to find the velocity as a function of the ratio $R=O / I$.

$$
V=\frac{A_{2}-A_{1} R}{B_{1} R-B_{2}}
$$

This rational function of $V=V(R)$ could also have been obtained by directly doing a nonlinear fit, but this would have required an iterative algorithm.

After the calibration constants are obtained, the data may be processed by using equation 13 for each data point to obtain a velocity time history. The power spectrum of the velocity fluctuations can also be calculated. Because of the high noise levels, it is necessary to use relatively long data records and process them using a technique such as described in the following section.

\section{Welch method of modified periodograms}

We used the Welch method of modified periodograms ${ }^{11}$ to calculate an estimate of the power spectrum of the velocity fluctuations. In this procedure, a long data record sampled at rate $f_{s}$ for time $T$ (total samples $=N=T f_{s}$ ) is subdivided into a number $K_{r}$ of smaller records (which may be overlapping), each of length $L$ samples. The modified periodograms of each sub-record are calculated using a data window; these individual periodograms are then averaged to obtain the estimate of the power spectrum. The frequency resolution of the resulting spectrum is thus $f_{s} / L$. By overlapping the segments by one half of their length, a near maximum reduction in the variance in the spectral estimate is achieved; the variance in the estimated spectrum is reduced by a factor of $11 / 9 K_{r}$ compared to the variance of the spectral estimate calculated directly from the original long data record.

\section{RESULTS}

Although the eventual application of this technique is the study of turbulent flows, the measurements reported here were all taken in the potential core of the subsonic free jet where the turbulence level should be quite low. This initial work 
in the free jet allowed the system to be evaluated for known flow conditions, where the flow velocity. density, and temperature could easily be determined using isentropic flow relations.

An example of a set of mean velocity measurements (each calculated using 100,000 samples taken at a $10 \mathrm{kHz}$ rate) is shown in figure 7 . The solid line is the velocity calculated from the measured total and ambient pressures using isentropic flow relations. The calibration constants described in the data processing section were obtained from data taken five days earlier. The rms deviation of the mean velocities from the isentropic values is $7.7 \mathrm{~m} / \mathrm{sec}$. This plot shows that the Fabry-Perot stabilization system is able to reproduce the correct interferometer alignment (locking to a preset fringe diameter before each measurement).

The distribution function of the counts from PMT 1, which are proportional to density, is shown for two flow velocities ( 22 and $284 \mathrm{~m} / \mathrm{sec}$ ) in figure 8 . The square of the standard deviation of the distribution (the variance) is very close to the mean, as expected for the Poisson statistics of the photoelectron counts. Also note that at the higher velocity, the density is greater because the temperature is reduced by the isentropic expansion.

The velocity distributions of two time history records are shown in figure 9 along with Gaussian fits. Since the measurements were taken in the potential core of the jet, the actual turbulence intensity is low and the fluctuation in the velocity is due almost entirely to the shot-noise. The measured values of velocity standard deviation, about $20-30 \mathrm{~m} / \mathrm{sec}$, agree with the predictions of the lower bounds calculations shown earlier. The use of the standard deviation as a measure of turbulence intensity can only be done for velocity fluctuations on the order of, or larger than the standard deviation due to the shot-noise. In this situation, since the shot-noise and velocity fluctuations are independent and add in quadrature, one can subtract the contribution of the shot-noise. However, we can see that this is much more attractive for higher velocity flows.

Finally, two examples of velocity power spectra calculated using the Welch method of modified periodograms described above (with $K=195$ overlapping segments of length $L=1024$ ) are shown in figure 10. For the low velocity flow $(22 \mathrm{~m} / \mathrm{sec})$ shown in Figure 10a, the spectrum is relatively flat, except for very low frequencies. For the higher velocity flow $(284 \mathrm{~m} / \mathrm{sec})$ shown in Figure $10 \mathrm{~b}$, the spectrum shows significant power in the frequency range $200-800 \mathrm{~Hz}$. This is most likely caused by flow fluctuations generated in the nozzle air supply line.

\section{CONCLUDING REMARKS}

An improved technique for obtaining dynamic gas density velocity measurements using molecular Rayleigh scattering was described. An analysis was presented that established a lower bound for measurement uncertainty of about $20 \mathrm{~m} / \mathrm{sec}$ for individual velocity measurements. The velocity was determined by analyzing the scattered light with a Fabry-Perot interferometer. The signals from three photomultiplier tubes were simultaneously recorded using photon counting at a $10 \mathrm{kHz}$ sampling rate and 10 second recording periods. Software and hardware interfaces were developed to allow computer control of all aspects of the experiment and data acquisition. Density and velocity measurements taken in a subsonic free jet were presented. The measured velocity standard deviation was close to the lower bound prediction. Velocity and density power spectra were obtained using the Welch method of modified periodograms. The stabilization system used for the Fabry-Perot interferometer allowed calibration data obtained one day to be used for data acquired five days later. This technique, which simultaneously measures time history of density and velocity, will be an important new tool for study of noise generation in turbulent flows.

\section{REFERENCES}

${ }^{1}$ Schaffar, M., "Direct measurements of the correlation between axial in-jet velocity fluctuations and farfield noise near the axis of a cold jet", J. Sound and Vibration, 64, pp. 73-83, 1979.

${ }^{2}$ Panda, J., and Seasholtz, R.G., "Measurements of shock structure and shock-vortex interaction in underexpanded jets using Rayleigh scattering", Phys. Fluids, 11, pp. 3761-3777, 1999.

${ }^{3}$ Seasholtz, R., and Panda, J., "Multiple point dynamic gas density measurements using molecular Rayleigh scattering", $18^{\text {th }}$ International Congress on Instrumentation in Aerospace Simulation Facilities Conference, Toulouse, France, June 1417, 1999. [also NASA TM-1999-209295].

${ }^{4}$ Seasholtz, R.G., and J. Panda, "Rayleigh Scattering Diagnostic for Simultaneous Measurements of Dynamic Density and Velocity", AIAA $38^{\text {th }}$ Aerospace Sciences Meeting, Reno, NV, AIAA2000-0642, 2000.

5 Seasholtz, R.G., and J. Panda, "Rayleigh scattering diagnostic for dynamic measurement of velocity and temperature", AIAA $37^{\text {h }}$ Aerospace Sciences Meeting, Reno, NV, AIAA-99-0641, 1999. 
${ }^{6}$ Tenti, G., Boley, C.D. and Desai, R.C., "On the kinetic model description of Rayleigh Brillouin scattering from molecular gases", Can. J. Phys. 52, pp. 285-290, 1974.

${ }^{7}$ Seasholtz, R.G., "High-speed anemometry based on spectrally resolved Rayleigh scattering", Fourth International Conference on Laser Anemometry, Cleveland, Ohio, 1991 [also NASA TM-104522].

${ }^{8}$ Seasholtz R.G. and Lock, J.A., "Gas temperature and density measurements based on spectrally resolved Rayleigh-Brillouin scattering", NASA Langley Measurement Technology Conference, Hampton, VA, 1992.

${ }^{9}$ Whalen, A.D., Detection of Signals in Noise, Academic Press, New York, pp. 324-231, 1971.

${ }^{10}$ Vaughan, J.M., The Fabry Perot Interferometer, History, Theory, Practice and Applications, Adam Hilger, Bristol, pp. 89-112, 1989.

${ }^{11}$ Welch, P.D., "The use of fast Fourier transform for the estimation of power spectra: A method based on time averaging over short, modified periodograms", IEEE Trans. on Audio and Electroacoustics, AU-15, pp. 70-73, 1967. 


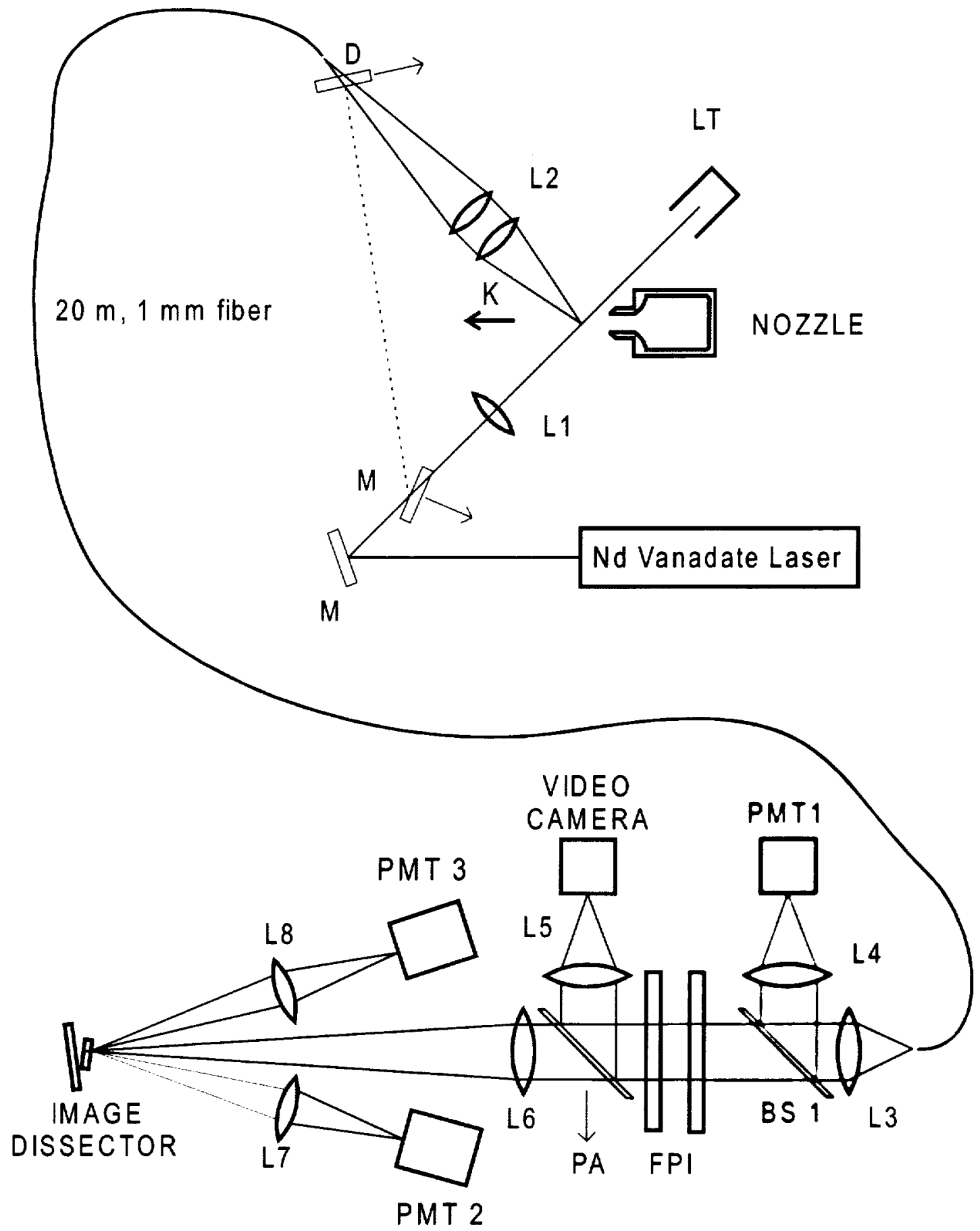

Fig. 1 - Layout of Rayleigh scattering system 


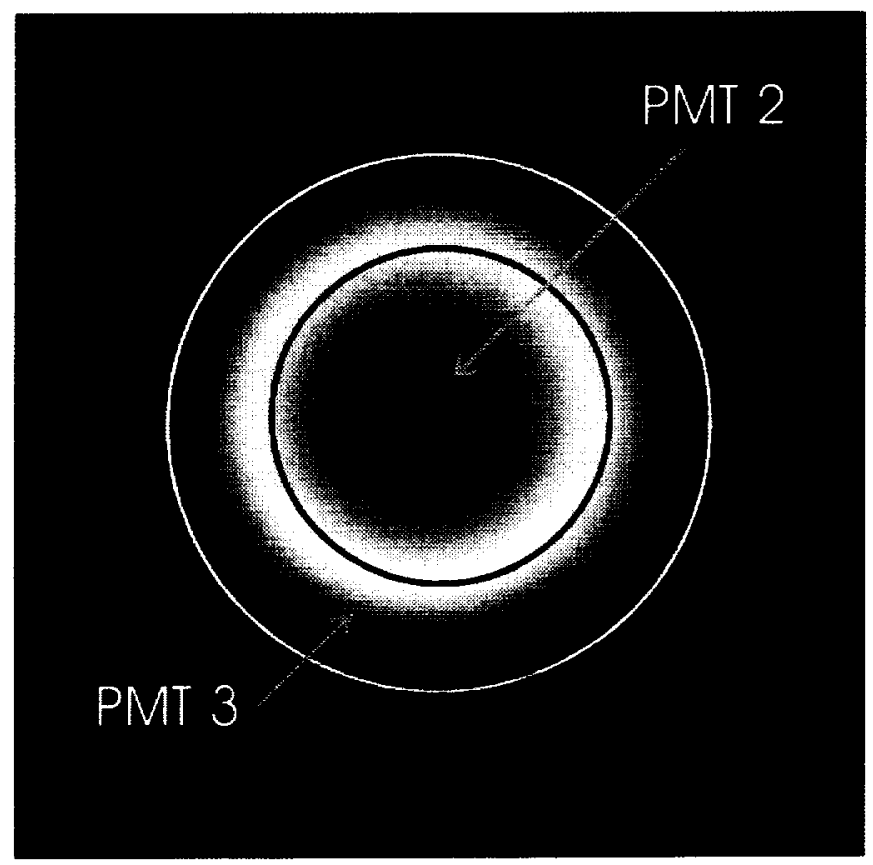

Fig. 2 - Fabry-Perot interferometer fringe showing two regions where light is directed to PMT 2 (INNER) and PMT 3 (OUTER). (a)

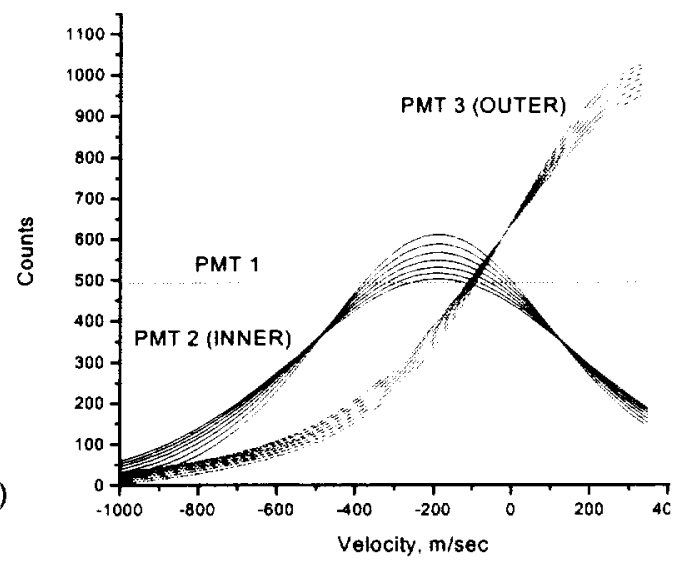

(b)

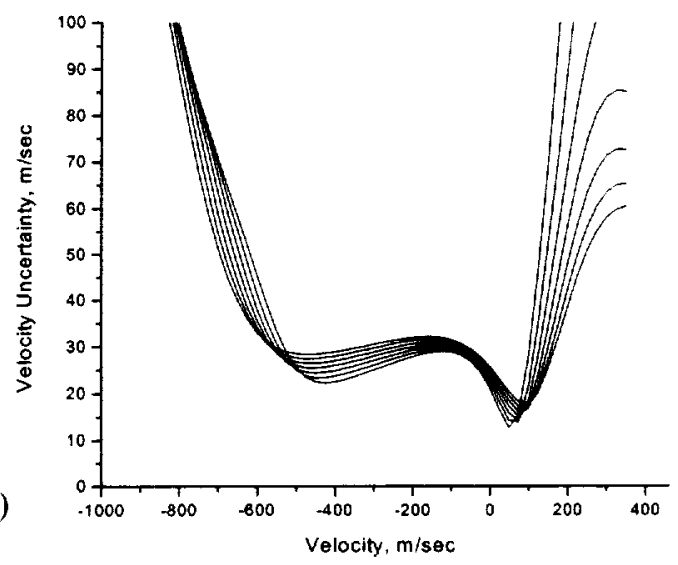

(c)

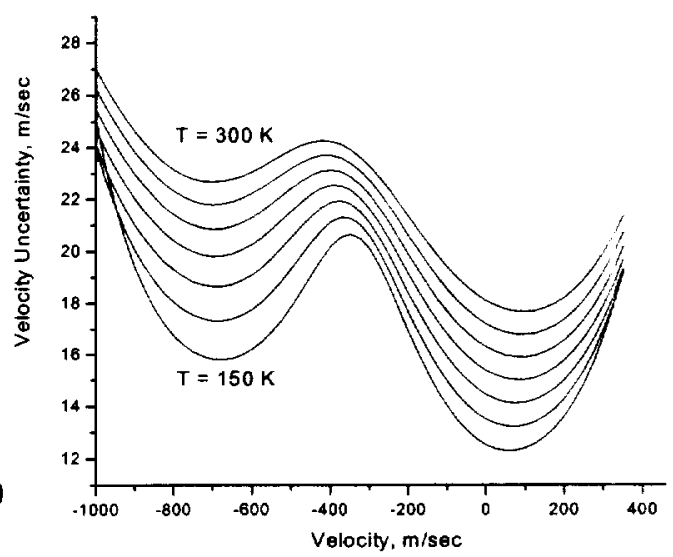

Fig. 3 (a) Number of counts for PMT 1, PMT 2 (INNER), and PMT 3 (OUTER) as function of velocity for zero velocity fringe order $n_{o}=0.10$; (b) Lower bound for velocity uncertainty for unknown temperature; (c) Lower bound for velocity uncertainty for known temperature. 

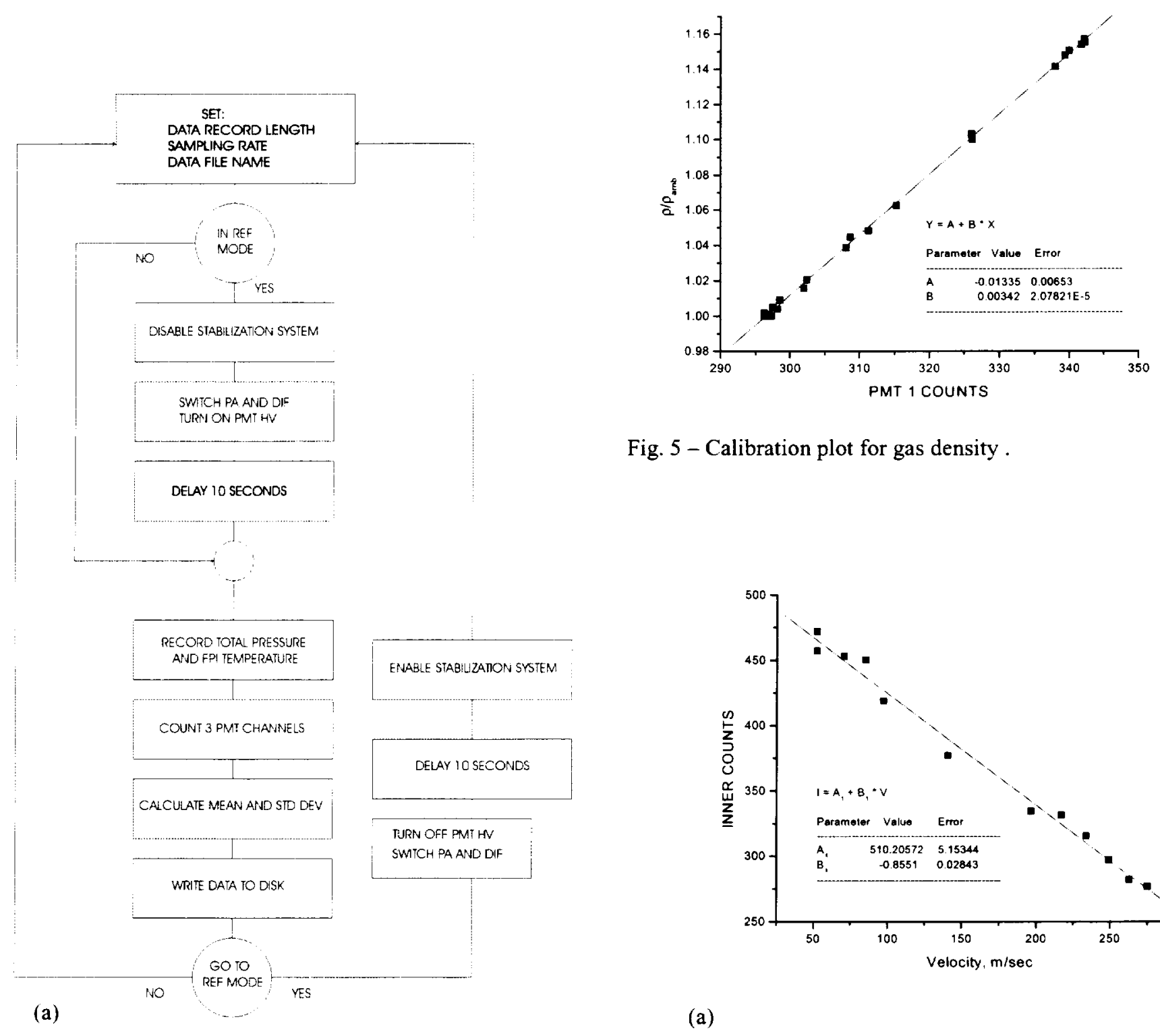

Fig. 5 - Calibration plot for gas density .

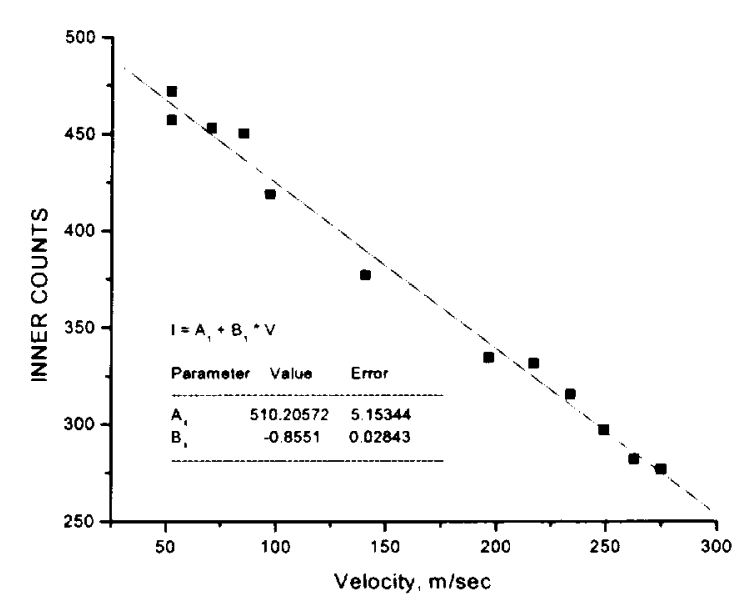

(a)

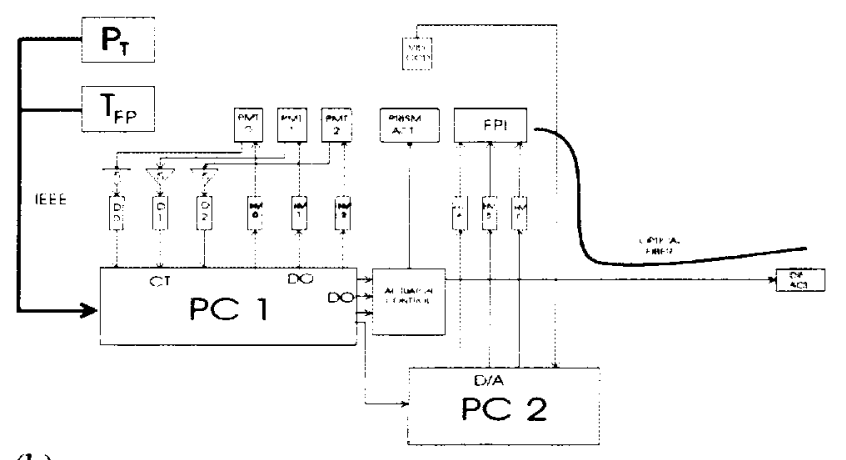

(b)

Fig. 4 - Experiment control and data acquisition system (a) Flow chart of system operation; (b) Block diagram 


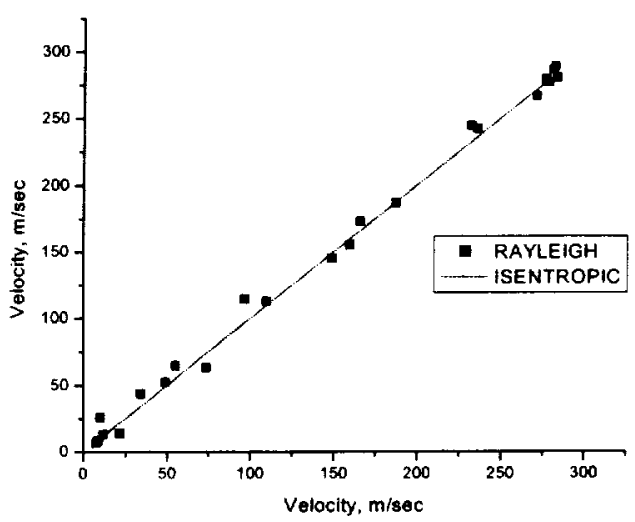

Fig. 7 - Velocity obtained from Rayleigh scattering measurements and from isentropic flow relations.

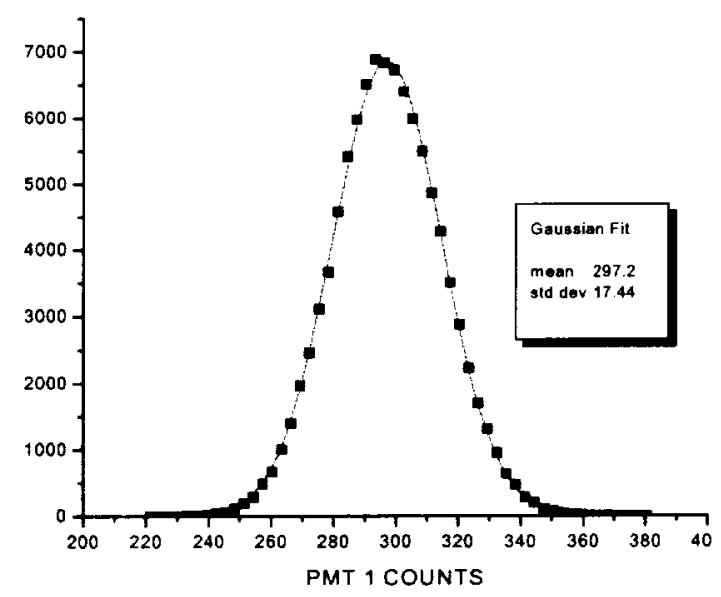

(a)

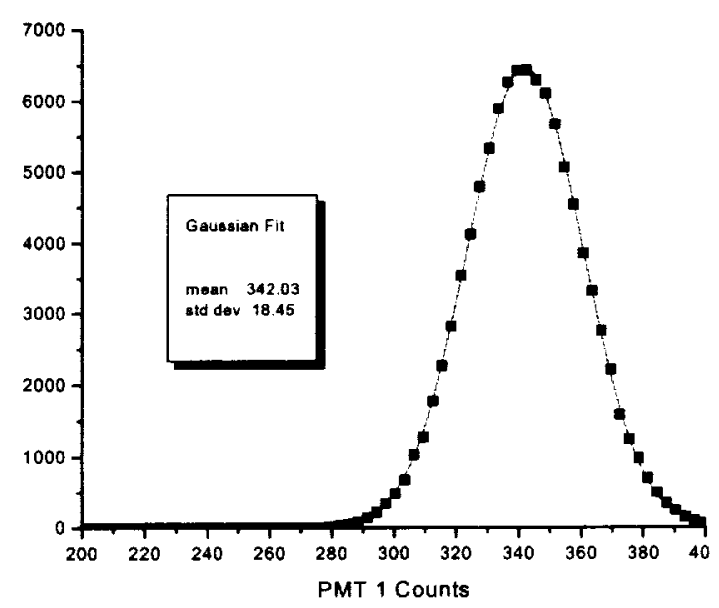

(b)

Fig. 8 - Distribution of PMT 1 counts (representing density) for mean flow velocity of $22 \mathrm{~m} / \mathrm{sec}$ (a) and $284 \mathrm{~m} / \mathrm{sec}$ (b).
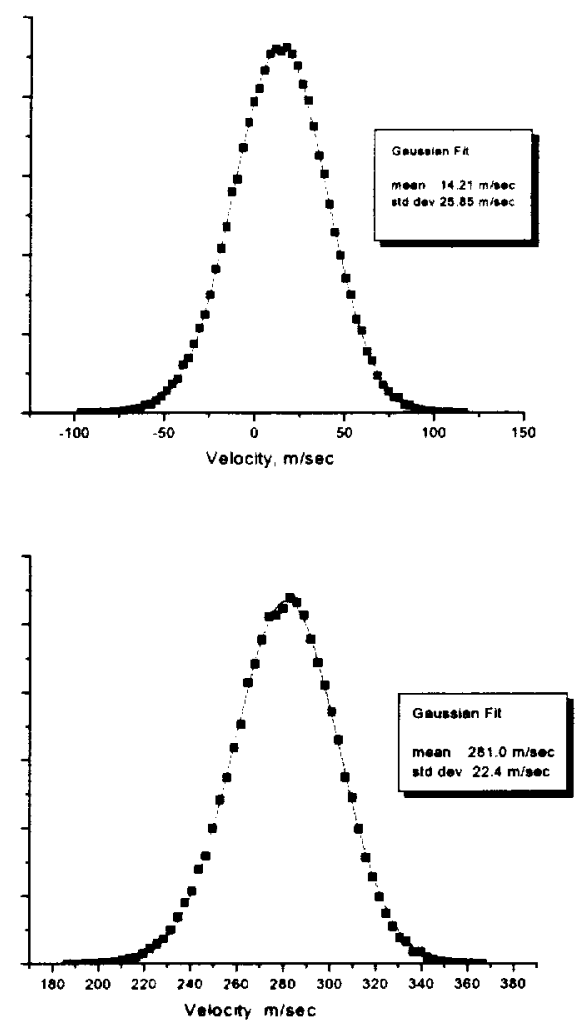

Fig. 9 - Velocity distribution function of mean flow $22 \mathrm{~m} / \mathrm{sec}$ (a) and $284 \mathrm{~m} / \mathrm{sec}$ (b).
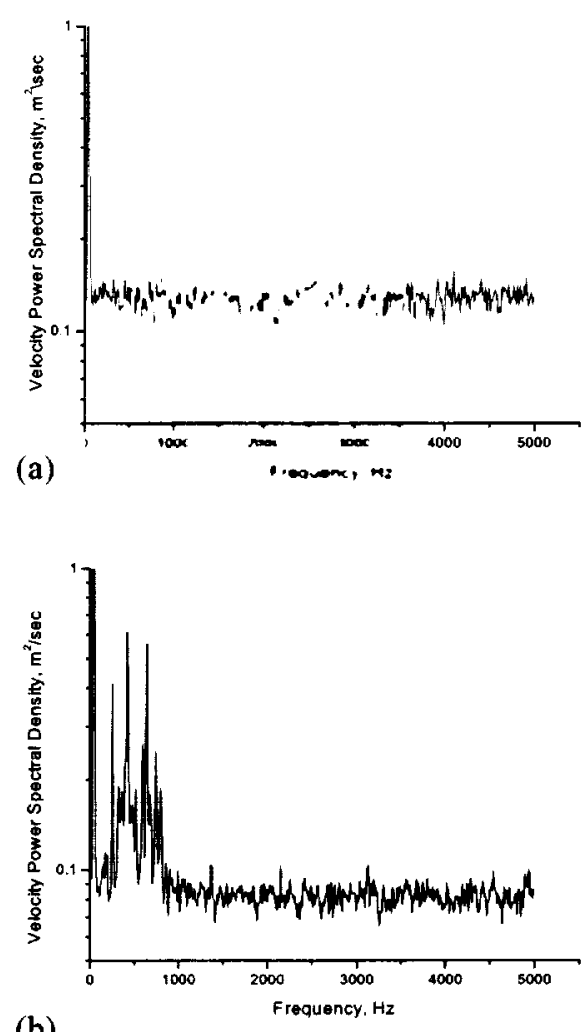

(b)

Fig. 10 - Power spectrum of velocity fluctuations for mean velocity $=22 \mathrm{~m} / \mathrm{sec}$ (a) and $284 \mathrm{~m} / \mathrm{sec}(\mathrm{b})$. 


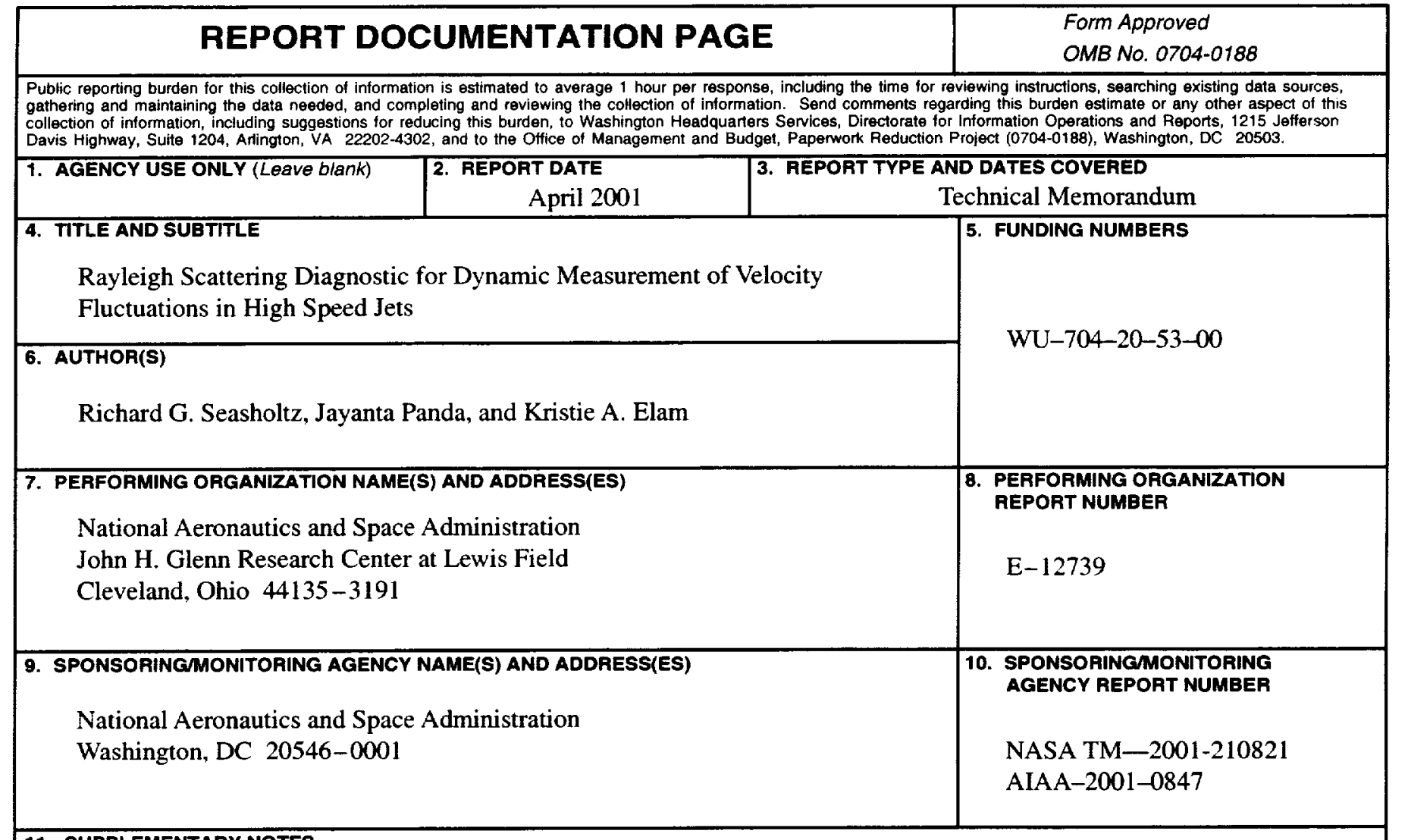

11. SUPPLEMENTARY NOTES

Prepared for the 39th Aerospace Sciences Meeting and Exhibit sponsored by the American Institute of Aeronautics and Astronautics, Reno, Nevada, January 8-11, 2001. Richard G. Seasholtz, NASA Glenn Research Center; Jayanta Panda, Ohio Aerospace Institute, 22800 Cedar Point Road, Brook Park, Ohio 44142; and Kristie A. Elam, Akima Corporation, Fairview Park, Ohio 44126. Responsible person, Richard G. Seasholtz, organization code 5520, 216-433-3754.

12a. DISTRIBUTIONAVAILABILITY STATEMENT 12b. DISTRIBUTION CODE

Unclassified - Unlimited

Subject Category: 35

Distribution: Nonstandard

Available electronically at http://gltrs.grc.nasa.gov/GLTRS

This publication is available from the NASA Center for AeroSpace Information, 301-621-0390.

13. ABSTRACT (Maximum 200 words)

A flow diagnostic technique based on the molecular Rayleigh scattering of laser light is used to obtain dynamic density and velocity data in a high speed flow. The technique is based on analyzing the Rayleigh scattered light with a FabryPerot interferometer used in the static, imaging mode. An analysis is presented that established a lower bound for measurement uncertainty of about $20 \mathrm{~m} / \mathrm{sec}$ for individual velocity measurements obtained in a $100 \mu \mathrm{sec}$ time interval. Software and hardware interfaces were developed to allow computer control of all aspects of the experiment and data acquisition. The signals from three photomultiplier tubes were simultaneously recorded using photon counting at a $10 \mathrm{kHz}$ sampling rate and 10 second recording periods. Density and velocity data, including distribution functions and power spectra, taken in a Mach 0.8 free jet are presented.

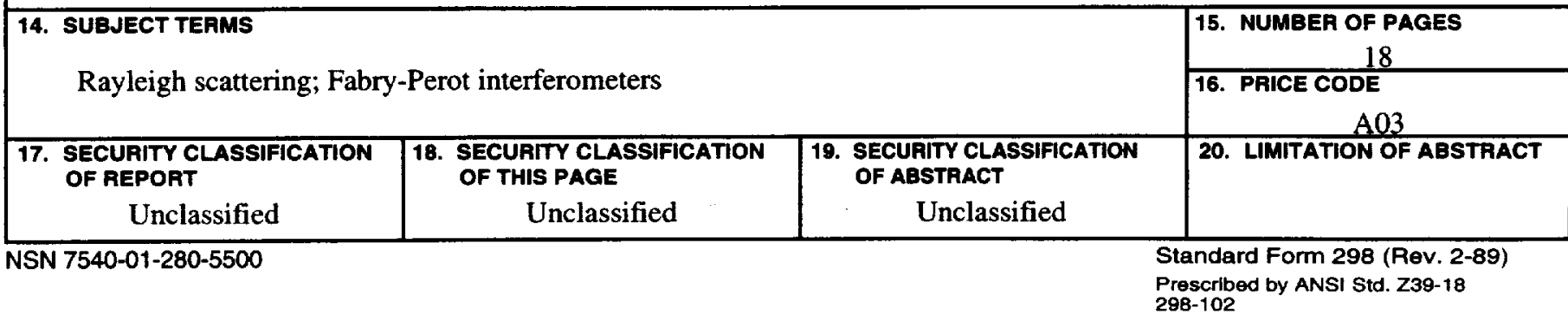

